\title{
Kinesin-7 CENP-E regulates chromosome alignment and genome stability of spermatogenic cells
}

\author{
Zhen-Yu She $\mathbb{1}^{1,2}$, Kai-Wei Yu $\mathbb{D}^{1,2}$, Ning Zhong ${ }^{1,2}$, Yu Xiao ${ }^{1,2}$, Ya-Lan Wei ${ }^{3,4}$, Yang Lin ${ }^{1,2}$, Yue-Ling $\mathrm{Li}^{1,2}$ and
} Ming-Hui Lu, ${ }^{1,2}$

\begin{abstract}
Kinesin-7 CENP-E is an essential kinetochore motor required for chromosome alignment and congression. However, the specific functions of CENP-E in the spermatogenic cells during spermatogenesis remain unknown. In this study, we find that CENP-E proteins are expressed in the spermatogonia, spermatocytes, and the elongating spermatids. CENP-E inhibition by specific inhibitor GSK923295 results in the disruption of spermatogenesis and cell cycle arrest of spermatogenic cells. Both spermatogonia and spermatocytes are arrested in metaphase and several chromosomes are not aligned at the equatorial plate. We find that CENP-E inhibition leads to chromosome misalignment, the spindle disorganization, and the formation of the aneuploidy cells. Furthermore, the inhibition of CENP-E results in the defects in the formation of spermatids, including the sperm head condensation and the sperm tail formation. We have revealed that kinesin-7 CENP-E is essential for chromosome alignment and genome stability of the spermatogenic cells.
\end{abstract}

\section{Introduction}

The genome stability of eukaryotes relies on accurate segregation of chromosomes during cell division, including mitosis and meiosis ${ }^{1}$. Chromosome alignment and kinetochore-microtubule attachment are essential for faithful chromosome segregation ${ }^{2}$. Chromosome instability results in aberrant chromosome numbers (namely aneuploidy) in the daughter cells ${ }^{3,4}$. Defects in chromosome alignment are closely related with aneuploidy and birth defects ${ }^{1}$. Detail mechanisms of meiotic cell division, particularly in male mammals, remain largely unknown.

Correspondence: Zhen-Yu She (zhenyushe@fjmu.edu.cn)

'Department of Cell Biology and Genetics, The School of Basic Medical Sciences, Fujian Medical University, Fuzhou, Fujian 350122, China

${ }^{2}$ Key Laboratory of Stem Cell Engineering and Regenerative Medicine, Fujian Province University, Fuzhou, Fujian 350122, China

Full list of author information is available at the end of the article

These authors contributed equally: Zhen-Yu She, Kai-Wei Yu

Edited by: N. Barlev
Centromere-associated protein-E (CENP-E) regulates chromosome alignment and mediates the kinetochoremicrotubule attachment ${ }^{5-7}$. CENP-E is a member of plusend-directed kinesin-7 subfamily, and is required for the congression of pole-proximal chromosomes ${ }^{8-10}$. Depletion of CENP-E in HeLa cells leads to mitotic arrest with misaligned chromosomes ${ }^{11-13}$. CENP-E mediates the kinetochore attachment and the tension on the centromeres $^{13-15}$. During cell cycle, CENP-E peaks at G2/M phase and locates at the outer kinetochore plate of chromosomes in prometaphase ${ }^{6,16}$. CENP-E promotes kinetochore-microtubule attachment during chromosome congression ${ }^{17}$. CENP-E is a large kinesin motor with a 230-nm flexible coiled coil, which facilitates chromosome capture and microtubule stabilization ${ }^{18-20}$. CENP-E plays a role in the metaphase-to-anaphase transition. CENP-E interacts with kinetochore proteins, including Bub1, BubR1, and Mps1, to mediate spindle assembly checkpoint $^{21-26}$.

\section{(c) The Author(s) 2020}

(c) (i) Open Access This article is licensed under a Creative Commons Attribution 4.0 International License, which permits use, sharing, adaptation, distribution and reproduction cc) in any medium or format, as long as you give appropriate credit to the original author(s) and the source, provide a link to the Creative Commons license, and indicate if changes were made. The images or other third party material in this article are included in the article's Creative Commons license, unless indicated otherwise in a credit line to the material. If material is not included in the article's Creative Commons license and your intended use is not permitted by statutory regulation or exceeds the permitted use, you will need to obtain permission directly from the copyright holder. To view a copy of this license, visit http://creativecommons.org/licenses/by/4.0/. 
The ablation of CENP-E in HeLa cells leads to chromosome misalignment with mono-oriented chromosomes at the spindle poles ${ }^{13,27,28}$. Deletion/inhibition of CENP-E results in metaphase arrest with several misaligned chromosomes and a prolonged cell cycle ${ }^{13}$. Deletion of CENP-E in mice shows chromosome missegregation and early embryo death ${ }^{5,26}$. CENP-E heterozygous $\left(C E N P-E^{+/-}\right)$mice show increased aneuploidy and tumor formation ${ }^{4,29}$.

Genetic deletion of CENP-E $\mathrm{E}^{5,26}$, the absence of CENP-E at kinetochores ${ }^{14}$, and the inhibition of CENP-E's motor activity ${ }^{18,30}$ suggest that CENP-E is essential for the faithful chromosome segregation. CENP-E regulates the chromosome alignment and spindle assembly checkpoint during meiosis $\mathrm{II}^{1,31}$. In D. melanogaster oocytes, CENP-E is essential for the alignment and movements of homologous chromosomes at meiosis $\mathrm{I}^{32}$. However, the specific functions of CENP-E in male meiotic division remain obscure.

The small molecule GSK923295 is a specific allosteric inhibitor of CENP-E, which inhibits the release of inorganic phosphate and stabilizes CENP-E in a rigor microtubule-bound state $^{33-35}$. GSK923295 treatment results in mitotic arrest and chromosome misalignment in metaphase. In tumor tissues, the ratio of $4 \mathrm{~N}$ to $2 \mathrm{~N}$ nucleus increases significantly after GSK923295 treatment. GSK923295-medieated CENP-E inhibition results in chromosome misalignment, cell cycle arrest, apoptosis, and tumor regression ${ }^{35}$.

In this study, we have revealed the expression pattern of kinesin-7 CENP-E in mouse spermatogenic cells. CENP-E proteins are expressed in the spermatogonia and spermatocytes. CENP-E locates at the manchette of the elongating spermatids during spermatogenesis. We have found that CENP-E inhibition results in the disruptions in spermatogenic waves and metaphase arrest of the spermatogium and spermatocytes. The ablation of CENP-E leads to chromosome misalignment in spermatocytes both in vivo and in vitro, which then stimulates the formation of aneuploidy cells. CENP-E regulates chromosome alignment in meiosis of primary spermatocyte. In addition, we have revealed that CENP-E inhibition influences nuclear condensation and the structures of sperm flagellum. In summary, we have revealed that kinesin-7 CENP-E plays an essential role in chromosome alignment and spindle assembly in spermatocytes, which promotes chromosome integrity and genome stability of male gametes.

\section{Results}

CENP-E proteins are expressed in the spermatogenic cells and CENP-E inhibition disrupts the normal cycles of spermatogenesis

To study the expression pattern of CENP-E proteins in mouse spermatogenic cells, we examined the localization of CENP-E proteins in mouse testes using immunofluorescence (Figs. 1; S1). CENP-E proteins were expressed in the spermatogonia, spermatocytes, and elongating spermatids. CENP-E proteins located at the cytoplasm in spermatogenic cells (Fig. 1a). At stage II, CENP-E proteins were distributed in the spermatogonia. At stage $\mathrm{V}$, CENP-E signals reached peak in the step 15 elongating spermatids. At stage IX, CENP-E located at the manchette of step 9 spermatids. In the elongating spermatids, CENP-E located at the microtubules of manchette (Fig. 1a). Thus, CENP-E proteins are expressed in all spermatogenic cells, indicating that CENP-E may play a role in spermatogenesis.

To investigate the functions of CENP-E in male mouse gonads, we used a small molecule inhibitor GSK923295, which specifically inhibited the ATP release of CENP-E and locked CENP-E in a rigor microtubule-bound state ${ }^{34,35}$ (Fig. 1b). We injected the GSK923295 into the right testis of 6-week-old ICR mice at a final concentration of 0.5, 1, 2, 4, and $30 \mu \mathrm{M}$, respectively (Fig. S1a, b). We compared the reproductive systems in the control and the GSK923295 group (Fig. 1b, c). There was no difference in the long diameter of mouse testes (Fig. 1d). The ratios of testis weight/body weight among $0.5,1$, 2, and $4 \mu \mathrm{M}$ GSK923295 treated group were similar to the control (Fig. 1e, f). After $30 \mu \mathrm{M}$ GSK923295 inhibition, the testis weight increased slightly compared with the control (Fig. 1g). Taken together, GSK923295 is a useful tool for long-term inhibition of CENP-E in vivo. And CENP-E inhibition does not significantly influence the weight of mouse testes.

To further study the effects of CENP-E inhibition in spermatogenesis at the cellular level, we examined the cellular structure of spermatogenic cells in seminiferous tubules using HE staining. In control, all spermatogenic cells were arranged orderly in spermatogenic epithelium. The spermatogenic epithelial waves were normal (Fig. 2a). We found that seminiferous tubules of the GSK923295 group were disordered. The thickness of each seminiferous tubule became smaller. The arrangement of spermatogonia and spermatocytes in the seminiferous tubules were abnormal. Specific stages of spermatogenic epithelial waves could not be clearly staged (Figs. 2b, c; S1c). In several seminiferous tubules, sperm cells were severely deformed and the head of the sperms were usually round. The spermatogenic epithelium became thinner. The distribution of spermatids was scattered and a portion of spermatids resided inside the seminiferous tubules (Fig. 2b, c). In addition, several spermatogenic cells showed a vacuolar structure, which might be lipid droplets observed by transmission electron microscopy (Fig. S1d).

\section{CENP-E inhibition results in the metaphase arrest of the spermatocytes during meiosis I}

At the cellular level, we found that CENP-E inhibition led to various abnormalities in spermatogenic cells. After GSK923295 treatment, a portion of the spermatogonia 


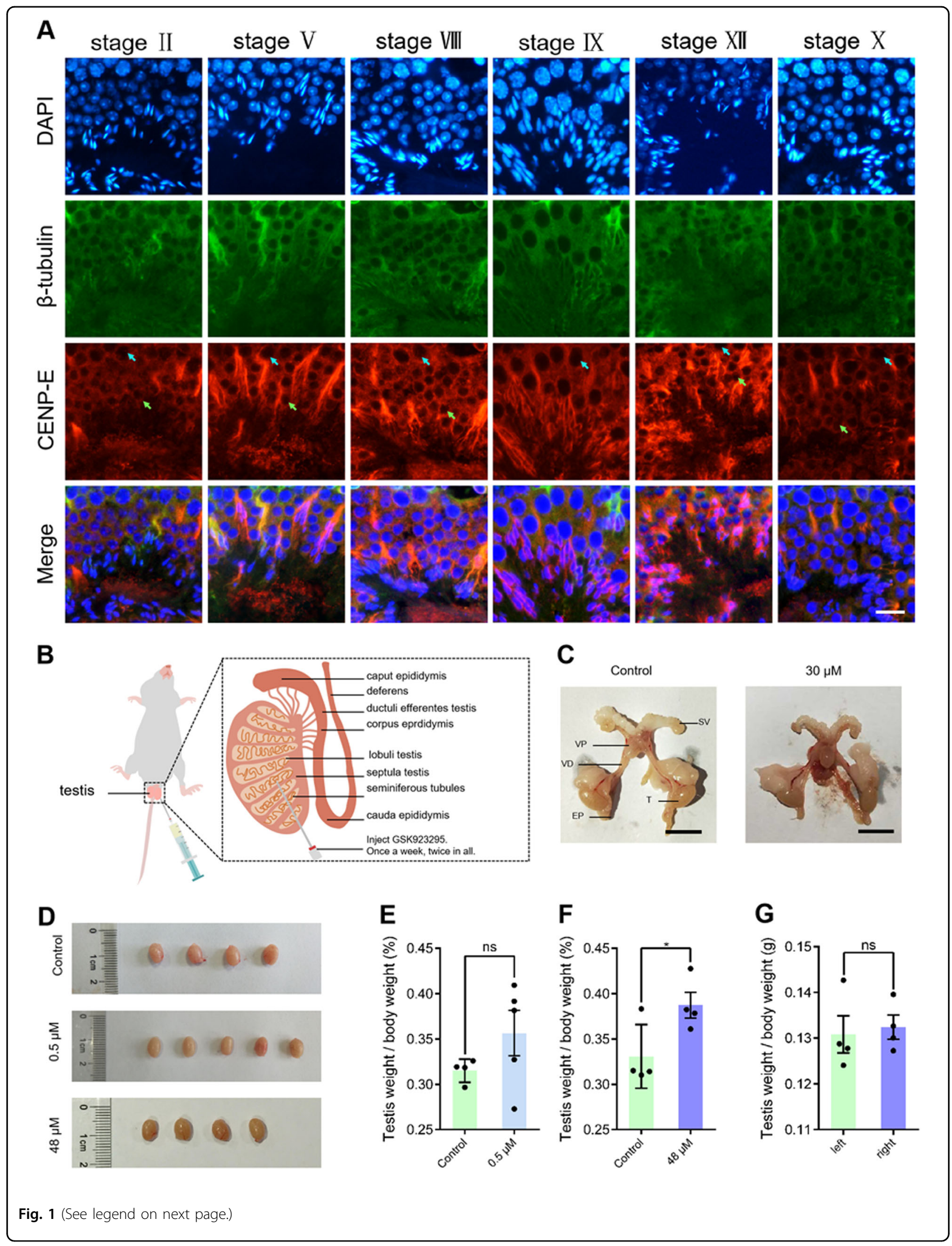


(see figure on previous page)

Fig. 1 The expression pattern of kinesin-7 CENP-E in mouse spermatogenic cells. a Immunofluorescence of CENP-E in mouse spermatogenic cells in testicular seminiferous tubules. DAPI was used to stain the nucleus. DAPI (blue), $\beta$-tubulin (green), and CENP-E (red). b Construction of a mouse model. Different concentrations of GSK923295 (14 $\mu \mathrm{M})$ were injected into the left testis of 8-week-old ICR mice to achieve inhibition of CENP-E. c Representative images of the male mouse reproductive system. Testis (T), epididymis (EP), seminal vesicles (SV), ventral prostate (VP), and vas deferens (VD). Scale bar, $1 \mathrm{~cm}$. d Representative images of mouse testes injected with $0.5 \mu \mathrm{M}, 30 \mu \mathrm{M}$ GSK923295. $n=4$ or $n=5$ per group. Control: $0.79 \pm 0.03 \mathrm{~cm} ; 0.5 \mu \mathrm{M}: 0.82 \pm 0.02 \mathrm{~cm} ; 30 \mu \mathrm{M}: 0.78 \pm 0.02 \mathrm{~cm}$. e-g Ratios of testis weight/body weight of testes after injected with $0.5 \mu \mathrm{M}$ and $30 \mu \mathrm{M}$ GSK923295. Mean values \pm SEM were shown. ns, $p>0.05,{ }^{*} p<0.05,{ }^{* *} p<0.01$ and ${ }^{* * *} p<0.001$. See also Fig. S1

were arrested in metaphase during mitosis. Several chromosomes failed to align at the equatorial plate in metaphase. Notably, we found that a significantly increased number of primary spermatocytes were arrested in metaphase I. In GSK923295 treated spermatocytes, several chromosomes were not aligned at the equatorial plate in metaphase (Fig. 2b). After CENP-E inhibition for 2 weeks, metaphase-arrested spermatocytes were a common phenotype. The primary spermatocytes were arrested in metaphase I with misaligned chromosomes away from the equatorial plate. These results indicate that CENP-E regulates chromosome alignment and cell cycle progression of primary spermatocytes.

To further investigate the cell populations in mouse testes, we digested the testes and stained the cells with propidium iodide (PI) for flow cytometry. In control, all the spermatogenic cells were divided into four groups according to the amounts of DNA content, including the haploid cells (spermatids and sperms, $1 \mathrm{~N}$ ), the diploid cells (spermatogonia and primary spermatocytes, $2 \mathrm{~N}$ ), the tetraploid cells (dividing primary spermatocytes, $4 \mathrm{~N}$ ), and a small portion of aneuploidy cell $(2-4 \mathrm{~N})$. After the injection of GSK923295 into mouse testes for 2 weeks, the ratios of the diploid cells increased from $8.17 \%$ to $9.93 \%$ in mouse testes (Fig. 3a-e). These results indicate that an increase in the ratios of spermatogonia and primary spermatocytes.

To study the long-term effects of GSK923295 inhibition, we injected GSK923295 into the abdomen of mice. In the intraperitoneal injection group for 10 times every 2 days, we observed a decrease in the proportion of haploid cells and a significant increase in tetraploid cells (Fig. $3 \mathrm{f}-\mathrm{j}$ ). These results suggest that the long-term inhibition of CENP-E leads to a decrease in the numbers of spermatids and dividing primary spermatocytes (Fig. 3). We also found that the number of spermatogenic cells between 2 and $4 \mathrm{~N}$ (aneuploidy cells) increased significantly. In the peak diagram, tetraploid cells also increased significantly (Fig. 3f), which indicated that cell cycle arrest and tetraploid cells could not divide normally. Taken together, the long-term inhibition of CENP-E leads to the metaphase arrest of primary spermatocytes and the formation of aneuploidy cells during spermatogenesis.

We found that the nuclei of spermatogonia and spermatocytes after CENP-E inhibition were slightly different from the control group (Fig. 4). The quantifications of chromatin mass density indicated that the heterochromatin increased in the nucleus of spermatogonia and spermatocytes (Fig. 4a-c, e, f). In spermatocytes, the organization of the nucleolus, including the fibrillar center, the dense fibrillary component and granular component, was slightly influenced after CENP-E inhibition (Fig. 4d-f).

\section{CENP-E inhibition leads to chromosome misalignment and spindle defects in dividing spermatocytes}

To study the underlying causes of chromosomal aneuploidy and cellular abnormalities, we firstly selected the GC-2 spd cells as our model cells. The GC-2 spd (ts) cell line was established by stable transfection of mouse spermatocytes with the SV40 large $\mathrm{T}$ antigen gene. The GC-2 spd cells remained at the spermatocyte stage and could not differentiate ${ }^{36}$. Thus, we selected the GC-2 spd cell as our model cell line to study cell division of spermatocytes in vitro. We treated the GC-2 spd cells with $100 \mathrm{nM}$ siRNA and then cultured for 24 and $48 \mathrm{~h}$. We found that CENP-E knockdown resulted in chromosome misalignment in the GC-2 spd cells at metaphase (Fig. 5a). In $6 \%$ GC-2 spd cells, the ablation of CENP-E led to several chromosomes can not align at the equatorial plate (Fig. 5b). We also compared the spindle abnormalities in CENP-E-depleted GC-2 spd cells with the control group. We found that there was no significant difference in the control and siRNA groups (Fig. 5c). After CENP-E knockdown for $24 \mathrm{~h}$, a significantly increased number of cells showed chromosome misalignment and metaphase arrest (Fig. 5a-c).

We also treated HeLa cells with GSK923295 and then cultured for 3, 6, or $24 \mathrm{~h}$. After GSK923295 treatment for $3 \mathrm{~h}$, we observed that several chromosomes could not align at the equatorial plate and a portion of HeLa cells were arrested in metaphase (Fig. S3). The inhibition of CENP-E resulted in $82.06 \%$ of HeLa cells became round and arrested at metaphase compared with $7.20 \%$ in the control (Fig. S3a, c). Giemsa staining showed that GSK923295 treatment resulted in a decrease in the number of HeLa cells, indicating that CENP-E inhibition led to a longer duration of cell cycle (Fig. S3b). After inhibition of CENP-E, a number of HeLa cells showed metaphase arrest. In addition, there were 
A
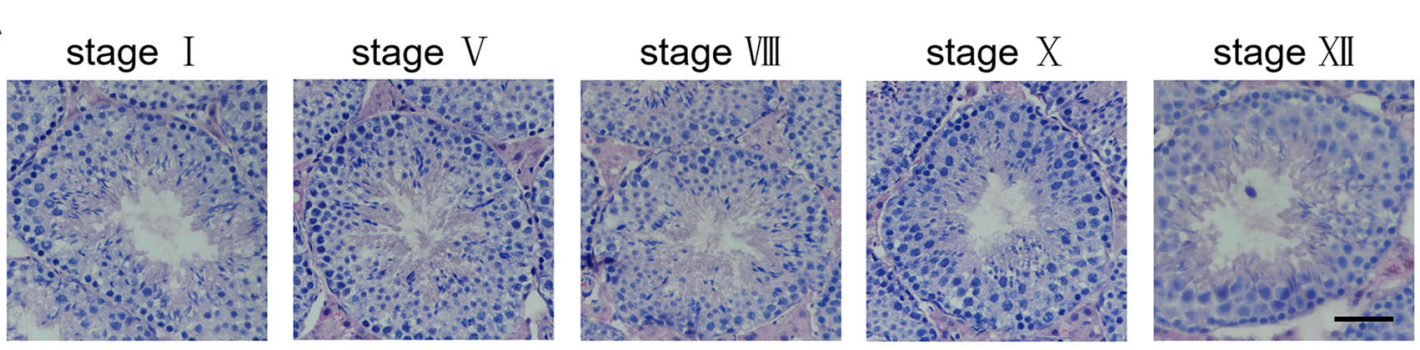

B
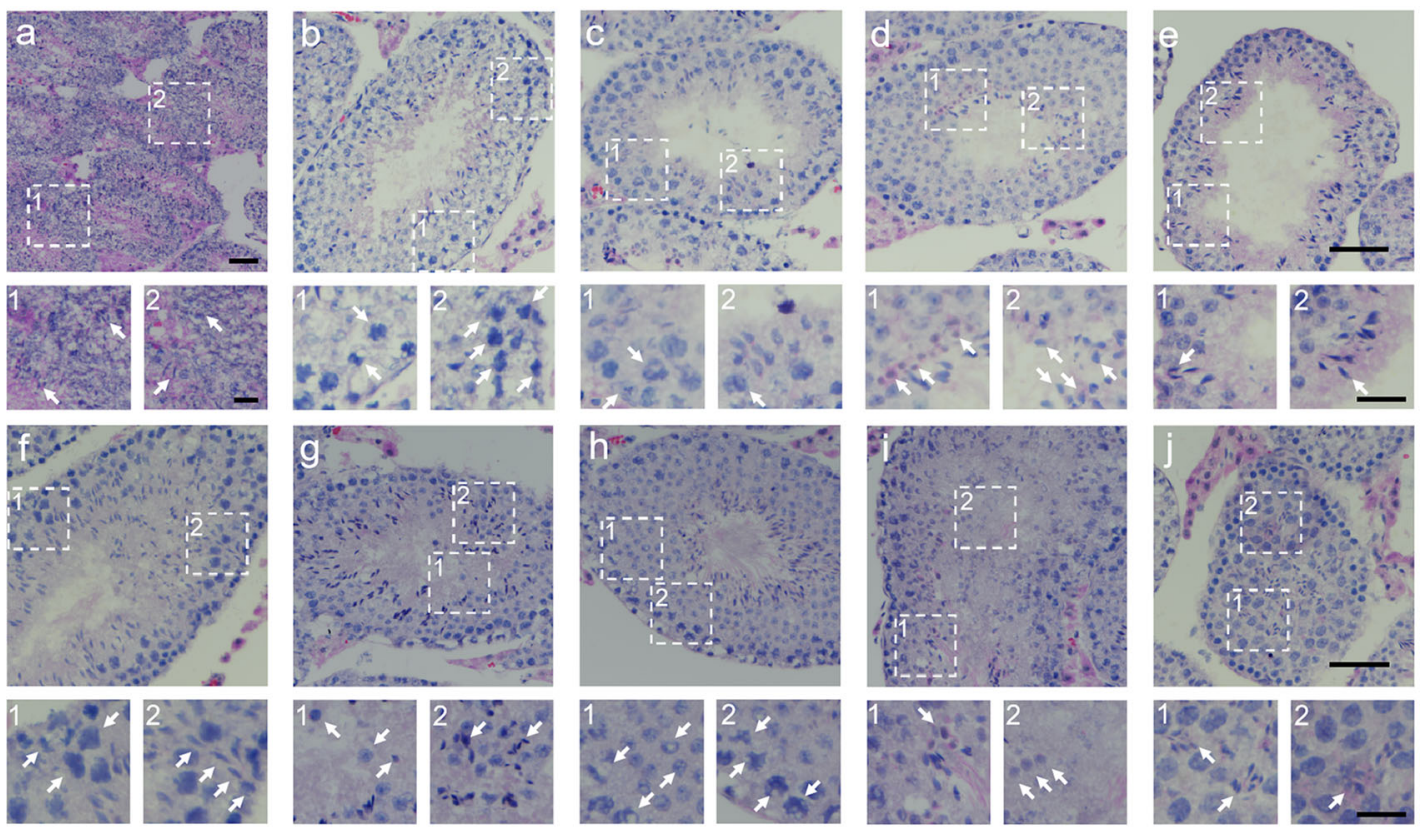

C
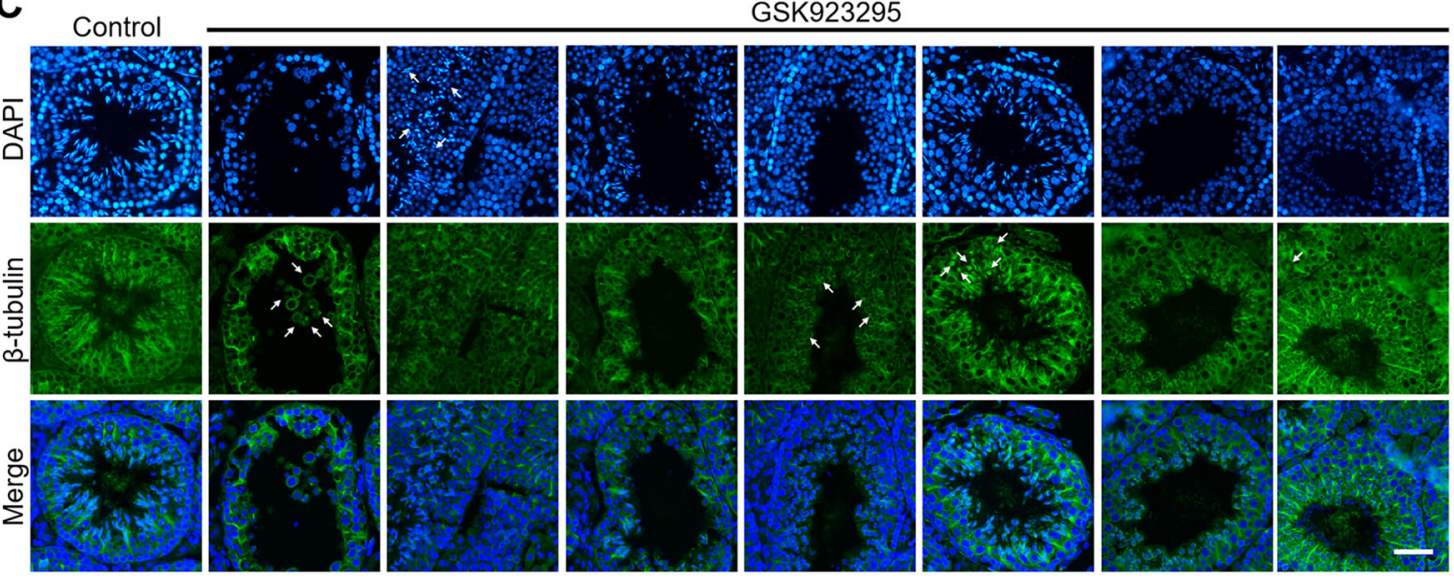

Fig. 2 CENP-E inhibition results in the testicular structural disorder and metaphase arrest of spermatocytes. $\mathbf{a}$, $\mathbf{b}$ Representative images of HE staining of the mouse testicular sections in the control (A) and GSK923295 (B) group. Scale bar, $50 \mu \mathrm{m}$. Relative stages were shown at the top of the panel. The enlarged images of spermatogenic cells were shown at the bottom of the panel. Scale bar, $20 \mu \mathrm{m}$. The arrows indicate the abnormal spermatogonia (c1), the arrested spermatocytes (b1, b2), and the dead sperms (d1). c Representative images of immunofluorescence of the mouse testicular sections. DAPI (blue) and $\beta$-tubulin (green). Scale bar, $50 \mu \mathrm{m}$. See also Fig. S2 


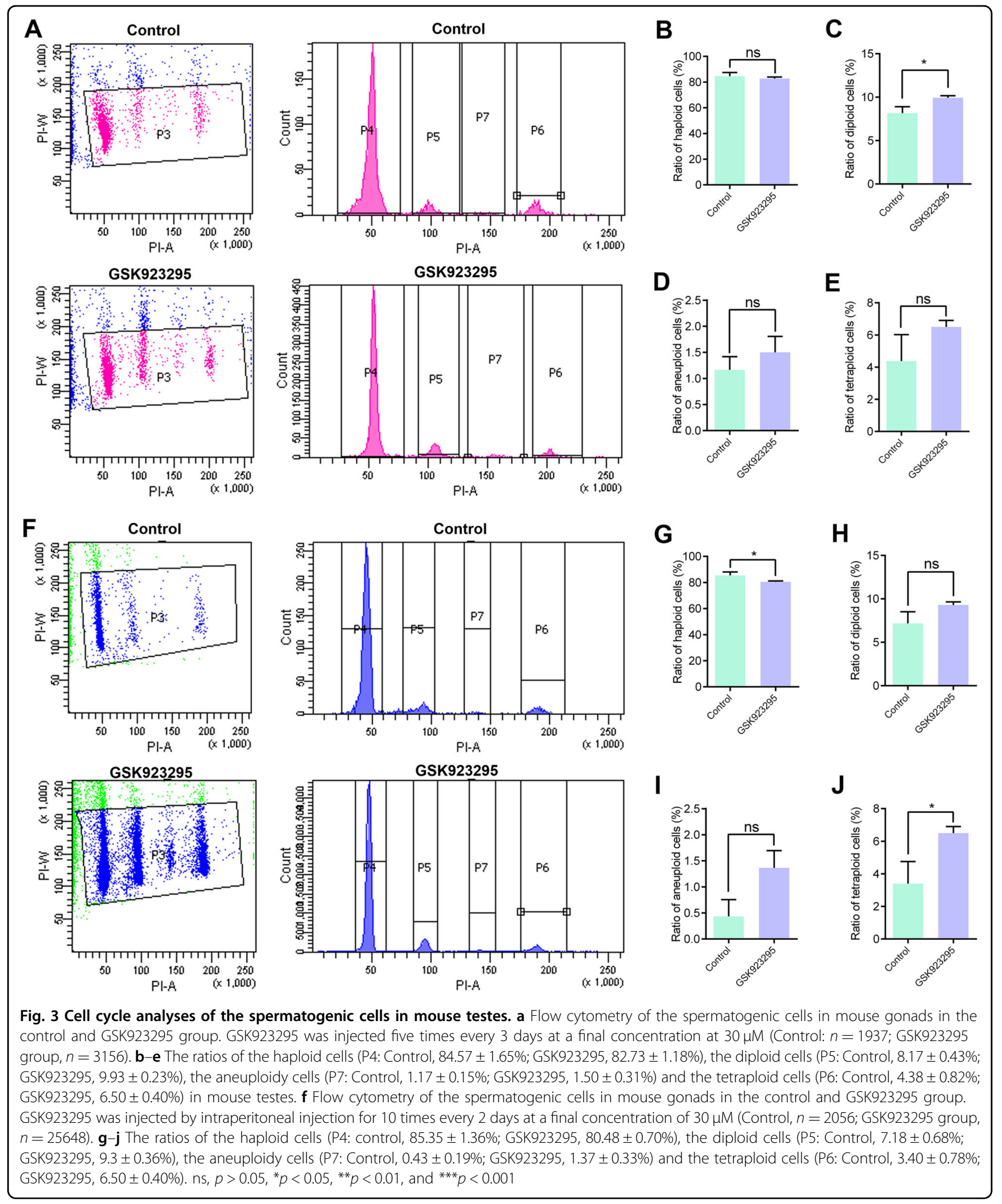

abnormalities in huge cells and multinucleated cells (Fig. S3d, e). In addition, we also found several abnormal spindle microtubule disorganization in $\mathrm{HeLa}$ cells, including the monopolar spindles, the multipolar spindles, the spindle protrusion and the curved spindles (Fig. S3f, i). These results indicate that CENP-E is essential for chromosome alignment in both cultured spermatocytes and HeLa cells. 


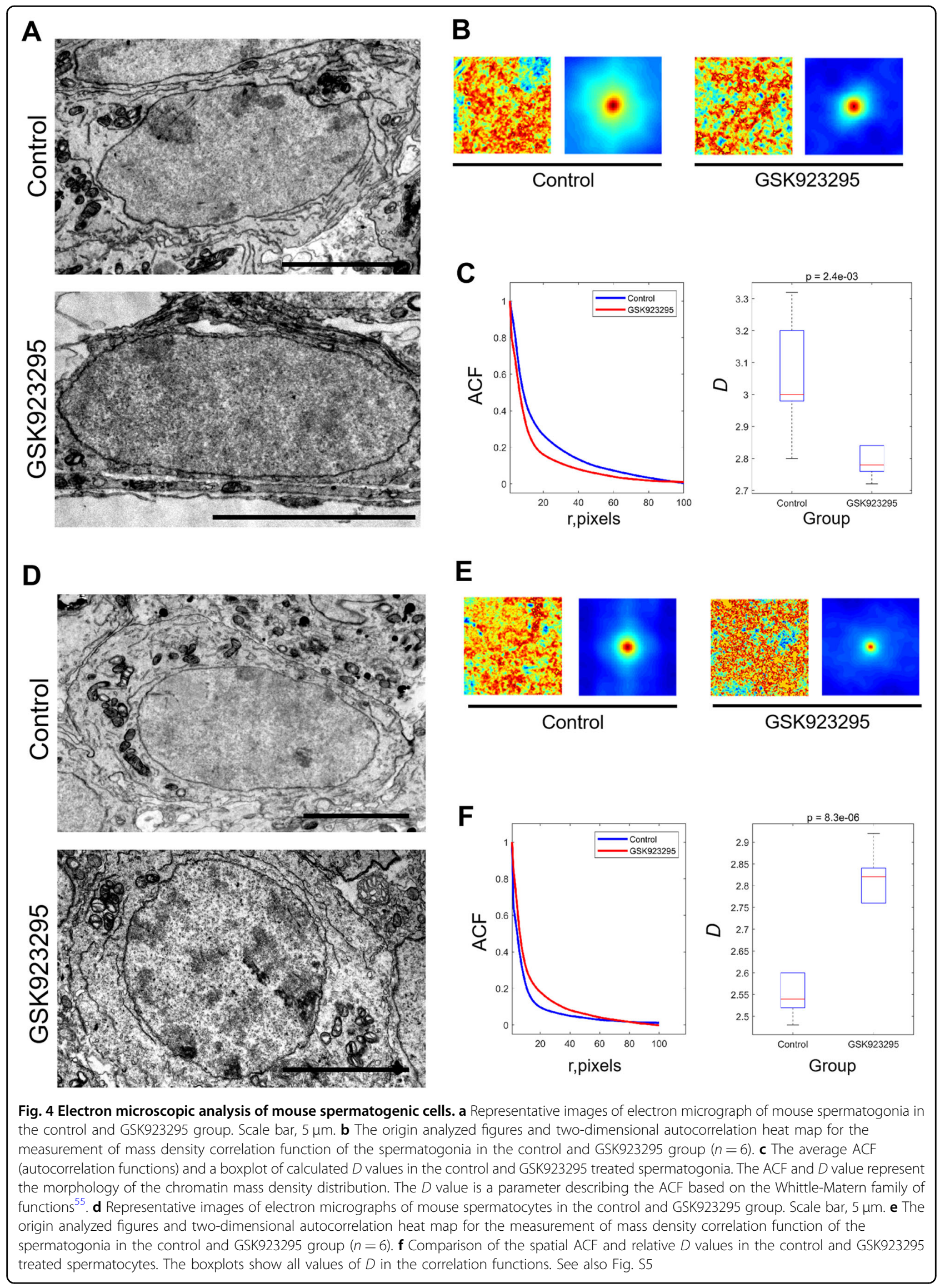

Official journal of the Cell Death Differentiation Association 


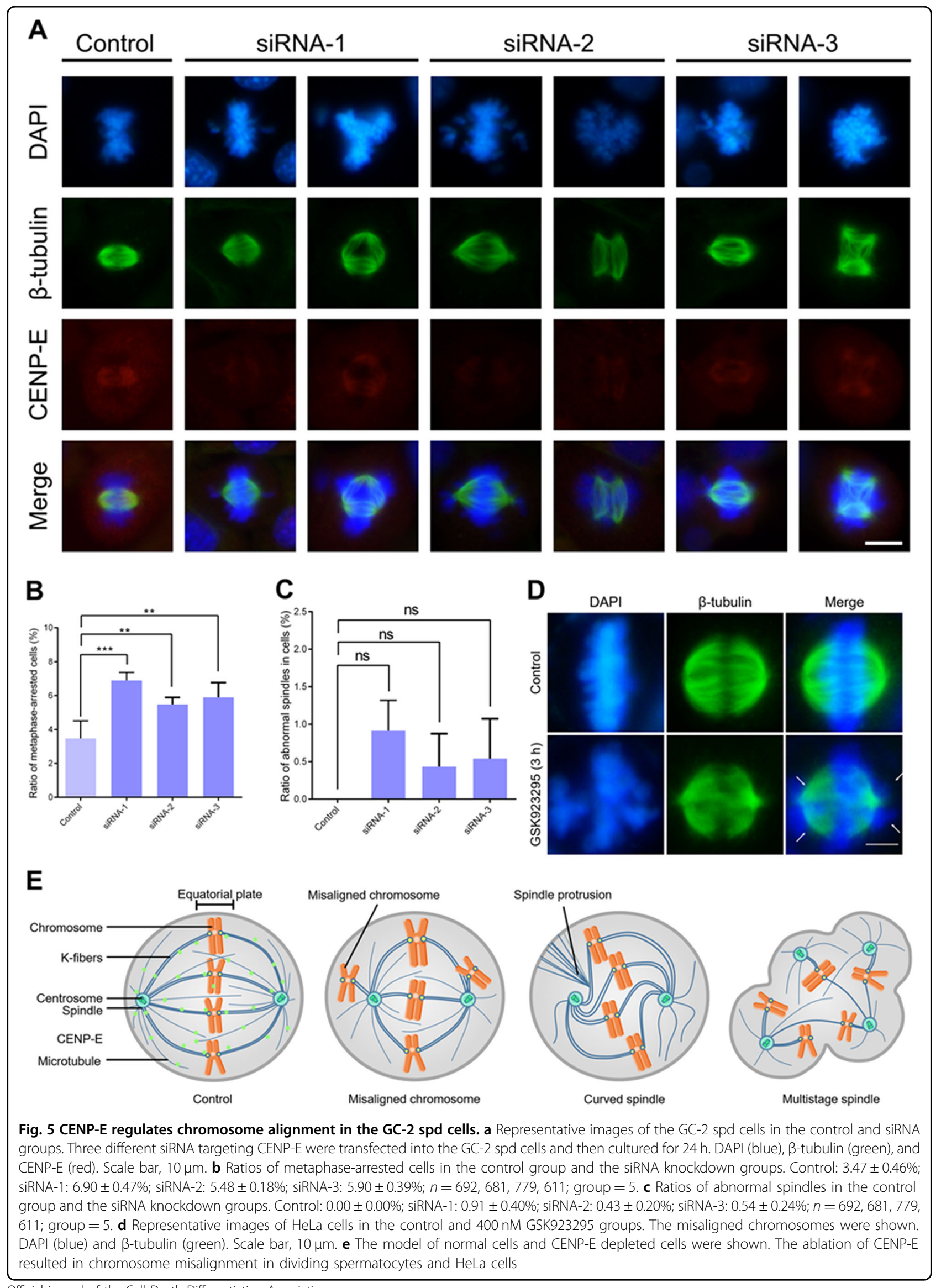

Official journal of the Cell Death Differentiation Association 
Kinesin-7 CENP-E proteins located at the regions of centromeres in the nucleus of the GC-2 spd cells in interphase (Fig. 6a). In prophase, CENP-E proteins located at the cytoplasm and spindle microtubules. In metaphase, all chromosomes were aligned at the equatorial plate and a portion of CENP-E proteins located at kinetochores (Fig. 6a-c). In anaphase, CENP-E proteins located at central spindle and the cytoplasm (Fig. 6a, c). To investigate specific roles of CENP-E in dividing spermatocytes, we examined the morphology of the GC-2 spd cells after GSK923295 treatment. Interestingly, we found that the staining of the nucleus in the GC-2 spd cells significantly changed compared with the control after Giemsa staining (Fig. 6d). Meanwhile, vacuolization abnormalities in the GC-2 spd cells were common after CENP-E inhibition (Fig. 6d). We found that a portion of chromosomes were not aligned at the equatorial plate after the siRNA-mediated knockdown of CENP-E in the GC-2 spd cells (Fig. 6e). In addition, a portion of GC-2 spd cells were round in interphase (Fig. 6f). The morphology of the GC-2 spd cells changed from flatten to fusiform (Fig. 6g).

We found that CENP-E inhibition resulted in several phenotypes, including chromosome misalignment in the metaphase arrested cells (Fig. 6h), the multinucleated cells (Fig. 6i), the abnormal nucleus (Fig. 6j), and the formation of micronuclei (Fig. 6k). We found that ratios of metaphase arrested GC-2 spd cells significantly increased to $1.58-4.20 \%$ in the presence of $400 \mathrm{nM}, 2 \mu \mathrm{M}$, and $6 \mu \mathrm{M}$ GSK923295 compared with $0.61 \%$ in the control (Fig. 6h). The ratios of multinucleated cells also significantly increased to $22.83-25.94 \%$ in the GSK923295 treated group compared with $9.50 \%$ in the control (Fig. 6i). Taken together, CENP-E inhibition results in metaphase arrest of dividing spermatocytes in vitro. The multinucleated cells and cells with micronuclei also increased after GSK923295 treatment, indicating that CENP-E inhibition led to the failure in chromosome segregation.

\section{CENP-E inhibition results in sperm head malformation and principal piece bending}

In order to study whether CENP-E inhibition influences the morphology and structure of mature spermatozoa, we squeezed the semen and stained the spermatozoa using HE staining (Fig. 7a). The abnormal sperms in the GSK923295 treated group significantly increased compared with the control. There were four kinds of sperm deformities, including the abnormal sperm head (Fig. 7b), the bent principal piece (Fig. 7c), the short tail malformation (Fig. 7d), and the bent midpiece (Fig. 7e). The ratios of these four kinds of malformation in sperms increased significantly compared with the control (Fig. 7b-e).

The long-term inhibition of CENP-E resulted in severe deformity of the mature spermatozoa in mice after the injection of GSK923295 for 2 weeks (Figs. 7; S4). We divided the sperm head malformations into six subtypes, including $69.23 \%$ of the irregular head sperms, $12.82 \%$ of the round head sperms, $7.69 \%$ of the triangular head sperms, $5.12 \%$ of the headless sperms, $2.56 \%$ of the large head sperms, and $2.56 \%$ of the double head sperms (Fig. 7f). These results indicate that CENP-E inhibition affects the morphology of the sperm head, the midpiece, the principal piece, and the endpiece.

We labeled $\beta$-tubulin to detect the structures of microtubules in the spermatozoa after CENP-E inhibition. We found that the microtubules of the flagella became discontinuous (Fig. 7g). Using electron microscopy, we found that the sperm head was relatively regular in the control group, but the nuclear shape was deformed and electron density of the nucleus was abnormal in GSK923295 group (Fig. 7h). In addition, the spermatids and spermatocytes in the control group had uniform nucleus, which contained homogenous chromatin (Fig. S5). However, CENP-E inhibition resulted in the irregularly distributed heterochromatin in the nucleus in spermatids and spermatocytes (Fig. S5b).

\section{Acute treatment of GSK923295 affects sperm maturation and causes sperm abnormality}

In order to illustrate whether acute treatment of GSK923295 influences the morphology of the mature spermatozoa, we performed a short-term inhibition of CENP-E in the mature spermatozoa. We aimed to find out the effects of CENP-E inhibition at the mature sperm stage. The epididymis of 6-month-old male mice was extracted and diluted with $0.1,0.5,2.5$, and $12 \mu \mathrm{M}$ GSK923295, respectively. The semen was cultured at $30^{\circ} \mathrm{C}$ for $4 \mathrm{~h}$ in vitro (Fig. 8). The results showed that in the acute GSK923295 treatment group, the number of spermatozoa with the bent principal piece increased to 26.80-44.09\% in the GSK923295 treated group compared with $13.68 \%$ in the control group (Fig. $8 \mathrm{a}-\mathrm{c}$ ). The round head sperms also significantly increased (Fig. 8d). In contrast, there was no significant difference in the number and proportion of spermatozoa among the separation of the head and tail, the bent midpiece, the abnormal head, and the short tail sperm (Fig. $8 \mathrm{e}-\mathrm{h}$ ).

These results suggest that short-term inhibition of CENP-E also leads to abnormal structure of sperm tail and a small number of round-headed spermatozoa. However, the short-term inhibition of CENP-E results in a less deformity of sperm head compared with the longterm treated group. This indicates CENP-E involves in all the process of spermatogenesis, including the formation of nucleus at early stage and maintenance of spermatozoa in the mature stage.

In addition, we examined apoptosis of spermatogenic cells after GSK923295 treatment using the TUNEL assays (Fig. S6). We did not observe a large number of the apoptotic 


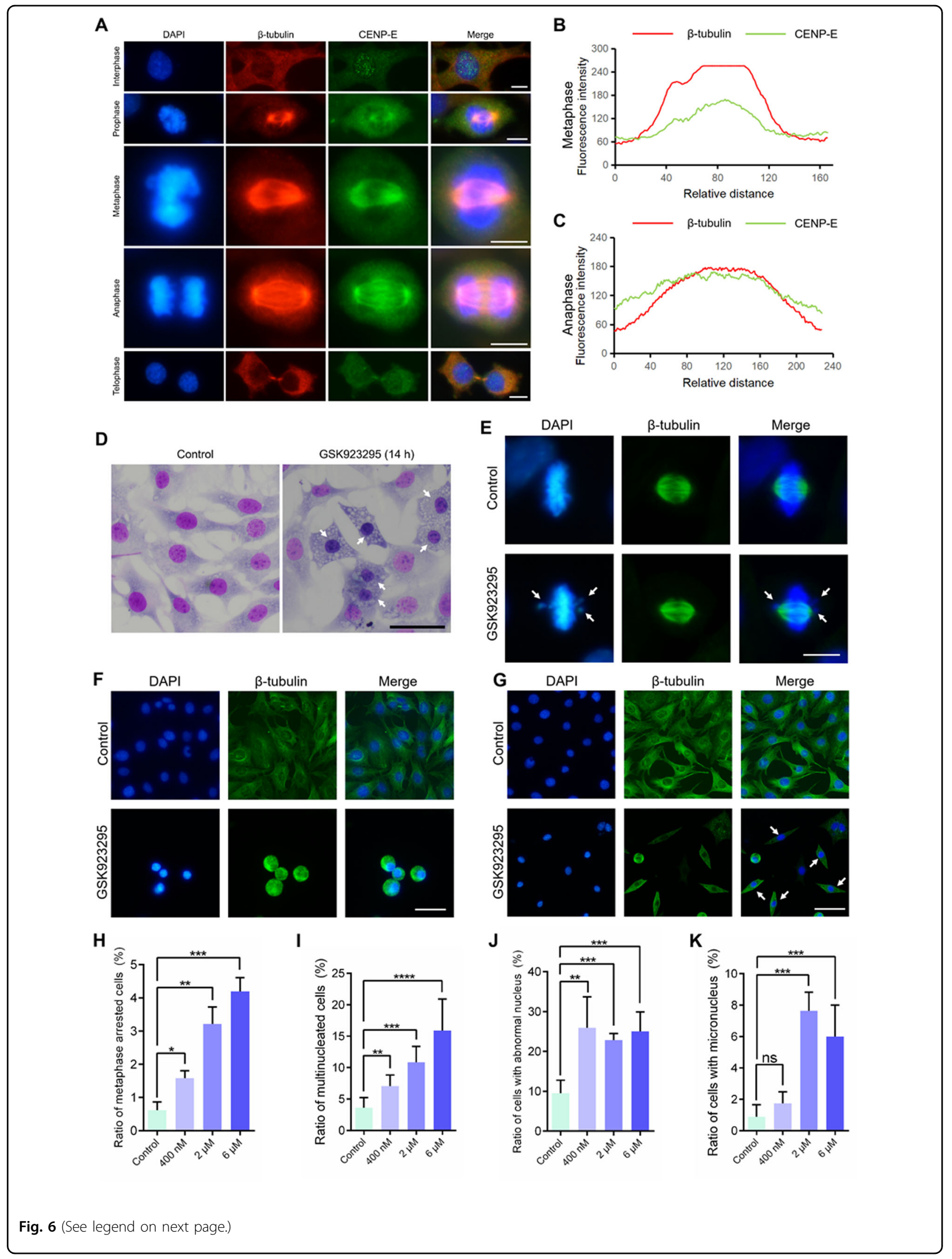


(see figure on previous page)

Fig. 6 CENP-E inhibition results in spindle disorganization and chromosome misalignment in the GC-2 spd cells. a Immunofluorescence of CENP-E in the GC-2 spd cells. DAPI (blue), CENP-E (green), and $\beta$-tubulin (red). Scale bar, $10 \mu \mathrm{m}$. b, c Image J analyses of the immunofluorescence intensities of indicated lines in GC-2 spd cells in metaphase and anaphase. $\mathbf{d}$ Giemsa staining of the GC-2 spd cells after incubated with $400 \mathrm{nM}$ GSK923295 for $14 \mathrm{~h}$. e Immunofluorescence of the GC-2 spd cells after incubated with $100 \mathrm{nM}$ siCENP-E-3 for $24 \mathrm{~h}$. DAPI (blue) and $\beta$-tubulin (green). Scale bar, $10 \mu \mathrm{m}$. f, $\mathbf{g}$ Immunofluorescence of the GC-2 spd cells after incubated with $400 \mathrm{nM}$ GSK923295 for $14 \mathrm{~h}$. DAPI (blue) and $\beta$-tubulin (green). Scale bar, $50 \mu \mathrm{m}$. $\mathbf{h}$ The ratios of metaphase arrested cells ( $\mathbf{h}$ Control, $0.61 \pm 0.25 \%$, group $=5, n=747 ; 400 \mathrm{nM}, 1.58 \pm 0.22 \%$, group $=5, n=630$; $2 \mu \mathrm{M}, 3.22 \pm 0.51 \%$, group $=3, n=364 ; 6 \mu \mathrm{M}, 4.20 \pm 0.42 \%$, group $=506, n=3)$. $\mathbf{i}$ The ratios of the multinucleated cells (i; Control, $9.50 \pm 1.63 \%$, group $=4, n=691 ; 400 \mathrm{nM}, 25.94 \pm 3.16 \%$, group $=6, n=300 ; 2 \mu \mathrm{M}, 22.83 \pm 0.83 \%$, group $=4, n=595 ; 6 \mu \mathrm{M}, 25.04 \pm 2.18 \%$, group $=5, n=488)$. $\mathbf{j}$ The ratios of cells with abnormal nucleus (j Control, $3.60 \pm 0.57 \%$, group $=4, n=691 ; 400 \mathrm{nM}, 7.07 \pm 0.78 \%$, group $=6, n=300 ; 2 \mu \mathrm{M}, 10.84 \pm$ $1.262 \%$, group $=4, n=595 ; 6 \mu \mathrm{M}, 15.88 \pm 2.25 \%$, group $=5, n=488$ ). $\mathbf{k}$ The ratios of cells with micronuclei ( $\mathbf{k}$ Control, $0.89 \pm 0.34 \%, g r o u p=4, n=$ $691 ; 400 \mathrm{nM}, 6.00 \pm 0.90 \%$, group $=6, n=300 ; 2 \mu \mathrm{M}, 1.74 \pm 0.33 \%$, group $=4, n=595 ; 6 \mu \mathrm{M}, 7.64 \pm 0.59 \%$, group $=5, n=488)$. See also Fig. S3

cells in cultured HeLa cells and the GC-2 spd cells (Fig. S6a), and in seminiferous tubules after 2 weeks inhibition (Fig. S6b). These results indicate that GSK923295 treatment does not result in the apoptosis of spermatogenic cells.

\section{Discussion}

Kinesin-7 CENP-E is expressed in mouse spermatogenic cells

CENP-E proteins locate at the centromeres in prometaphase of the cultured spermatocytes. In metaphase, CENP-E proteins locate at kinetochore fibers in dividing spermatocytes. During cytokinesis, CENP-E proteins locate at the central spindle and the midbody. In seminiferous tubules, CENP-E proteins are expressed in the spermatogenic cells (Fig. 1).

In human cells, CENP-E locates at the fibrous corona and the outer kinetochore plate ${ }^{37}$. In our study, we reveal that expression pattern of CENP-E in spermatocytes is similar to the previous results in somatic cells ${ }^{7,37,38}$, indicating a conserved role of CENP-E in mitosis and meiosis. CENP-E proteins are initially located at the outer kinetochore plate of homologous centromeres in late dikinesis/early prometaphase I during male mouse meiosis ${ }^{1,31,39}$. The inner centromere proteins MCAK and SGOL2 recruit CENP-E and BubR1 to the outer kinetochore plate of primary spermatocytes ${ }^{1}$. However, the specific functions of CENP-E in male mouse meiotic divisions remain largely unknown.

Our results indicate that CENP-E plays a role in male meiotic division. The small molecule inhibitor GSK923295 inhibits the ATPase activity of CENP-E and locks the motor domain in a rigor microtubule-bound state $^{35}$. Thus, GSK923295 is a unique tool to investigate the long-term consequence of CENP-E inhibition and developmental functions of CENP-E in this study.

\section{CENP-E is essential for the metaphase-to-anaphase transition in primary spermatocytes}

In somatic cells, CENP-E inhibition results in metaphase arrest and mitotic delay ${ }^{6}$. However, there are significant differences in the process of meiosis I and mitosis. For example, in meiosis I, homologous chromosomes undergo recombination and separation. However, in mitosis, sister chromatids separate and the cohesion breakdown ${ }^{40}$. Meiotic spindles also differ from the typical mitotic spindle ${ }^{27}$. Most studies of CENP-E are performed in somatic cells, while little information is available regarding the functions of CENP-E in mammalian meiosis and male spermatogenesis. In our study, we have revealed that CENP-E inhibition results in metaphase arrest of primary spermatocytes in meiosis I. This is the first evidence that CENP-E also involves in the regulation of the metaphase-to-anaphase transition of meiosis I.

Anti-CENP-E antibody injection into maturing mouse oocytes results in metaphase I arrest and metaphase II arrest $^{40}$, indicating that CENP-E might have a conserved role in female meiosis division. In our study, we have found that CENP-E inhibition by GSK923295 can result in the metaphase I arrest of primary spermatocytes both in vivo and in vitro. These results indicate that CENP-E is responsible for the metaphase-to-anaphase transition of primary spermatocytes in meiosis I.

In prometaphase, the unattached kinetochores produce a "wait anaphase" signal for the activation of spindle assembly checkpoint ${ }^{23}$. CENP-E interacts with the checkpoint components, including BubR1 and $\operatorname{Mad} 2^{11,41}$, and then participates in the establishment and maintenance of the spindle assembly checkpoint $^{21,23}$. Microtubule capture by CENP-E prevents the recruitment of Mad2 to kinetochores under tension and silences the BubR1-dependent spindle assembly checkpoint $^{3,25,42}$. Spindle assembly checkpoint is weakened at CENP-E-depleted kinetochores and a portion of chromosomes can not form the bipolar attachment $^{25,27,43}$. In HeLa cells, siRNA-mediated CENP-E inhibition leads to a delayed mitotic progression for $4 \mathrm{~h}$ due to misaligned chromosomes ${ }^{27,43}$. In our study, we have found that the metaphase arrested spermatocytes significantly increased both in vitro and in vivo (Fig. 2). Meanwhile, several chromosomes misaligned at the spindle poles. Whether metaphase arrest in meiosis I is caused by the activation of spindle assembly checkpoint remains to be clarified in future. 


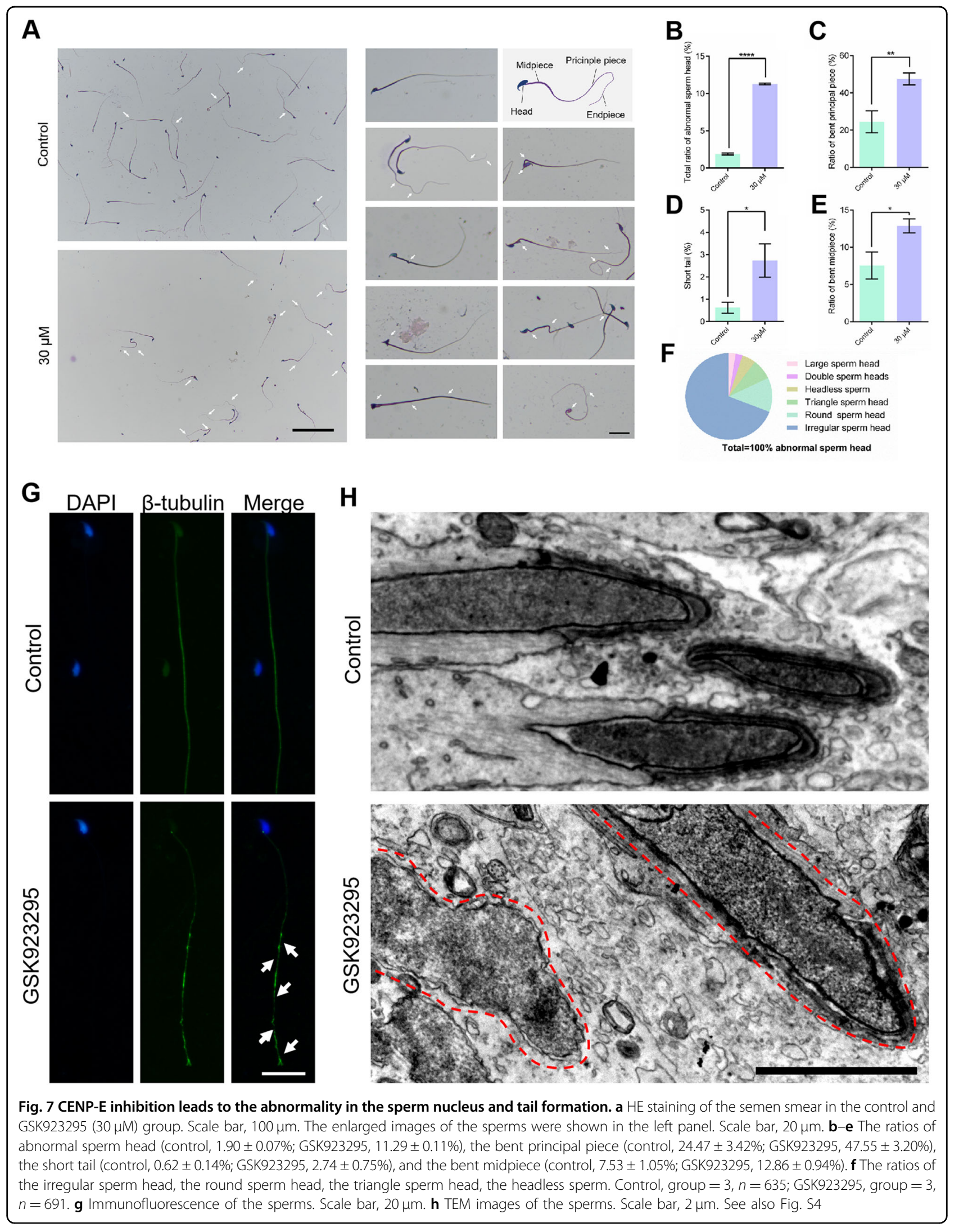




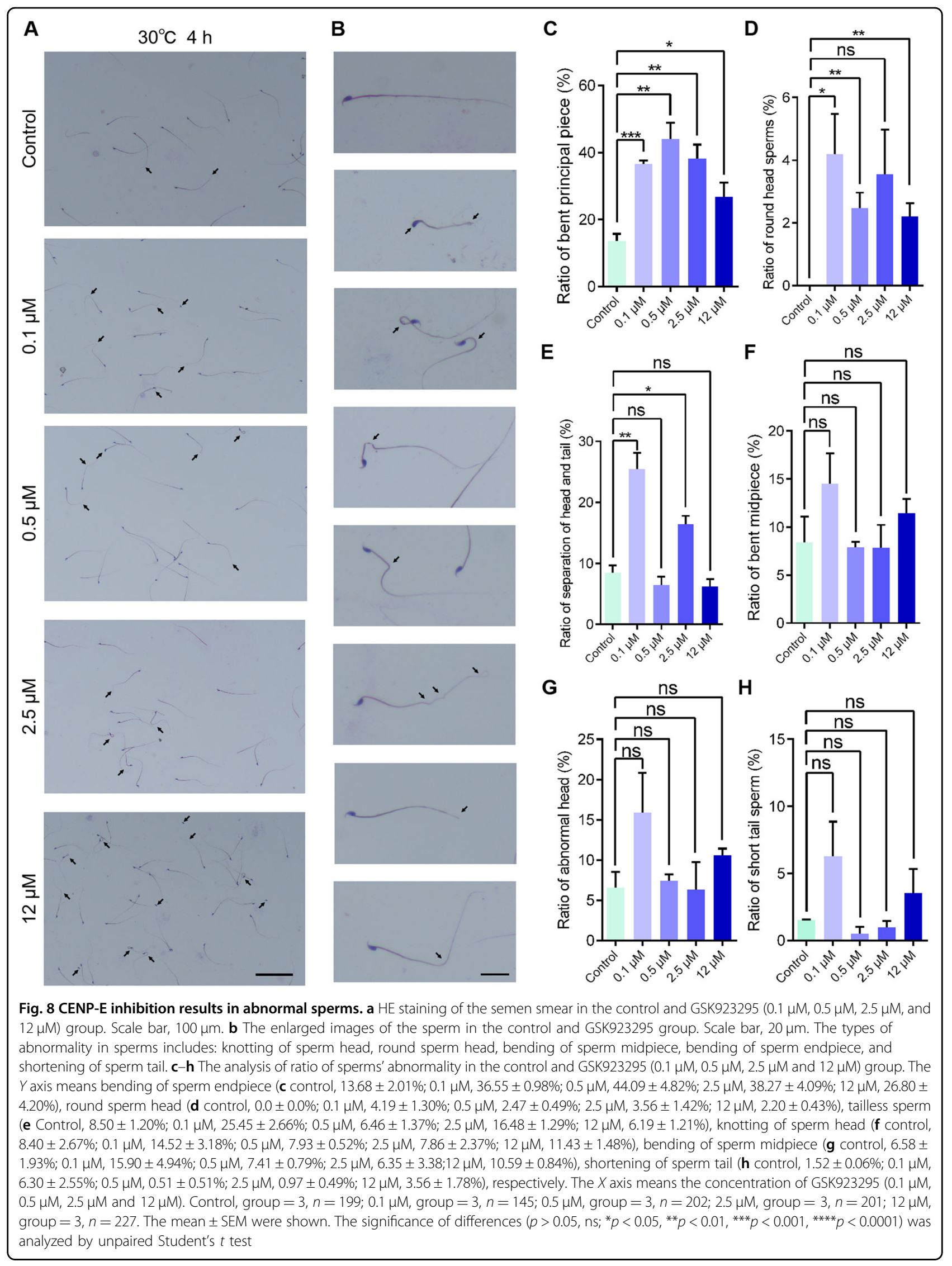




\section{CENP-E regulates chromosome alignment and spindle organization in spermatocytes}

Depletion of CENP-E leads to chromosome misalignment in Xenopus egg extracts ${ }^{27}, D$. melanogaster ${ }^{44}$, and vertebrate cultured cells ${ }^{12}$. CENP-E is a plus-end-directed kinetochore motor essential for chromosome alignment at the metaphase plate ${ }^{27,45}$. Kinetochore-microtubule interaction is regulated by CENP-E, which is crucial for chromosome alignment in the equator plate ${ }^{12}$. The ablation of CENP-E leads to significant numbers of monooriented chromosomes at the spindle poles ${ }^{12,14}$. CENP-E transports mono-oriented chromosomes to the spindle equator along mature kinetochore fibers ${ }^{46,47}$, and then stabilizes metaphase alignment ${ }^{48}$. CENP-E is a highly processive motor that generates forces ${ }^{49}$ and tethers the kinetochore to dynamic spindle microtubules ${ }^{8,19}$. Similarly, we have found that CENP-E inhibition results in chromosome misalignment in dividing spermatocytes (Fig. 6). These results suggest that CENP-E is also required for chromosome alignment in meiosis.

Chromosome missegreagtion and cytokinetic defects are caused by the defects in spindle assembly ${ }^{50}$. Previous studies have indicated that CENP-E depletion reduce kinetochore-microtubule attachment and tension ${ }^{14,51}$. The depletion of CENP-E leads to 50\% decrease in the density of the kinetochore fibers ${ }^{5,14}$. In our study, we have found that CENP-E inhibition results in multiple spindle defects, including curved spindles and microtubule disorganization in both HeLa and the GC-2 spd cells. In addition, CENP-E contains a 230-nm flexible coiled coil, which is involved in microtubule capture ${ }^{19}$. The elongated stalk domain is essential for the stabilization of kinetochore-microtubule attachment ${ }^{52}$. In mouse oocyte meiosis I, CENP-E depletion results in the polar chromosome displacement and the unstable kinetochoremicrotubule attachment ${ }^{53}$. CENP-E stabilizes BubR1 to promote meiosis I progression and regulates the chromosome bi-orientation by inhibition of chromosome drift to the spindle poles ${ }^{53}$. After chromosome congression, CENP-E severs as a processive bi-directional tracker for stable association between dynamic microtubule ends and kinetochores ${ }^{18}$. In male mouse meiosis, the misaligned chromosome at the spindle poles might be due to the microtubule capture by CENP-E is inhibited.

\section{CENP-E is responsible for genome stability of spermatogenic cells and the formation of sperms}

The stable propagation of genetic material relies on the chromosome congression at the spindle equator ${ }^{46}$. Genetic deletion of CENP-E in mice results in misaligned chromosomes in primary fibroblasts and high degree of aneuploidy, even to embryonic lethal ${ }^{5}$. The primary cultured cells and hepatocytes only proceed through several rounds of mitotic division ${ }^{5}$. CENP-E deletion reduces the number of kinetochore-associated microtubules, which leads to the lack of kinetochore-microtubule attachment ${ }^{5}$.

Heterozygous deletion of CENP-E in mice leads to gain or loss of intact chromosomes, but does not result in cytokinesis failure and tetraploidy ${ }^{29}$. In CENP-E deficient cells, the sister chromatids can not separate normally, which then increase the whole chromosome aneuploidy ${ }^{54}$. In our study, we have found that CENP-E inhibition in dividing spermatocytes leads to the increase of multinucleated cells and cells with micronuclei (Fig. 4). These results suggest that the gain and loss of intact chromosomes also exist in cell division of spermatocytes after CENP-E inhibition, which increases aneuploidy in daughter cells.

The inhibitor GSK923295 is reported to cause apoptosis in human HCC1954 breast carcinoma cells ${ }^{35}$. In our study, the TUNEL results indicate that few apoptotic cells exist and an increased numbers of dead cells after GSK923295 treatment. Previous studies have indicated that high chromosomal instability leads to cell death ${ }^{4}$. The increased number of dead cells in seminiferous tubules is caused by the missegreagtion of high numbers of chromosomes. In addition, abnormal structures and irregular shapes of the sperm midpiece, principal piece, and endpiece are mainly caused by microtubule instability after CENP-E inhibition during the spermatogenesis. We have found that both spermatogenesis and mature sperms are affected by the inhibition of CENP-E.

Our results address a long-standing question in cell division. Previous studies have demonstrated that CENP-E regulates chromosome congression and alignment in somatic cells and mediates spindle assembly checkpoint. In summary, our data provide the initial evidence that CENP-E is essential for chromosome alignment and congression in meiosis. CENP-E inhibition results in the defects in chromosome misalignment and congression, which leads to metaphase arrest in meiosis. CENP-E regulates the organization of the bipolar spindle to fulfill accurate chromosome alignment. CENP-E inhibition results in chromosome misalignment, chromosome missegreagtion, and the formation of aneuploidy cells. In addition, the depletion of CENP-E results in the defects in the spermatid formation, including the sperm head condensation and the sperm tail formation. Taken together, we have concluded that kinesin-7 CENP-E regulates the chromosome alignment in meiosis and maintains the genome stability of male spermatids.

\section{Materials and methods}

\section{Animals and ethics}

All animal experiments were conducted according to the Guide for the Care and Use of Laboratory Animals of Fujian Medical University. This study was approved by the Animal Care and Use Committee at Fujian Medical University (Protocol No. SYXK 2016-0007). 


\section{Drugs and reagents}

GSK923295 (MedChemExpress HY-10299) was dissolved in dimethyl sulfoxide (DMSO, Sigma-Aldrich D2650) and stored at a final concentration of $10 \mathrm{mM}$. Different concentrations of GSK923295 were diluted in $\mathrm{H}_{2} \mathrm{O}$ or $0.9 \%$ $\mathrm{NaCl}$ for CENP-E inhibition. For RNA interference in mouse GC-2 spd cells, the following siRNAs were synthesized: Negative Control, 5'-UUCUCCGAACGUGUCAC GUTTT-3'; siGapdh, 5'-GUAUGACAACAGCCUCAAGT T-3' (Mus musculus Gapdh, GenBank Accession No. NM_001289726.1); siCENP-E-1， 5'-GGAAGAAAGUCA AGAGGAATT-3'; siCENP-E-2, 5'-CUGCUGAACUGGAG AGAAATT-3'; siCENP-E-3, 5'-UGAAAGAGCAGGAGAA CAATT-3' (Mus musculus CENP-E, GenBank Accession No. NM_173762.4).

\section{Cell culture and treatment}

The GC-2 spd cell (ATCC No. CRL-2196) and HeLa cell (ATCC No. CCL-2) were cultured in DMEM/high glucose medium (Hyclone, Cat. SH30022.01) supplemented with $10 \%$ fetal bovine serum (Every green, Cat. 11011-8611), $4 \mathrm{mM}$ L-glutamine (Hyclone), $4.50 \mathrm{~g} / \mathrm{L}$ glucose (Hyclone), and $100 \mathrm{IU} / \mathrm{mL}$ Penicillin-Streptomycin (MP Biomedicals, Cat. 1670249). The cell lines was authenticated and tested for mycoplasma contamination. The identity of all cell lines are verified annually in our laboratory. The cells were cultured at $37^{\circ} \mathrm{C}$ and supplemented with $5 \% \mathrm{CO}_{2}$ in a humidified incubator (Thermo Fisher). For cell passage, 0.25\% Trypsin-EDTA (Gibco Cat. 25200-056) was used for digest. For CENP-E inhibition in cultured cells, GSK923295 were added into the culture medium as indicated and then cultured at $37^{\circ} \mathrm{C}$. For CENP-E siRNA interference, the siRNA, including negative control, siGapdh, siCENP-E-1, siCENP-E-2, and siCENP-E-3, was transfected into the GC-2 spd cells using Lipo 8000 transfection reagent according to the manufacturer's protocol (Beyotime Cat. C0533). The siRNA were incubated with Lipo8000 for $5 \mathrm{~min}$ and added into the culture medium at a final concentration at $100 \mu \mathrm{M}$. The GC-2 spd cells were then cultured for 24 and $48 \mathrm{~h}$ and harvested for subsequent analyses.

\section{Cryo-section and immunofluorescence}

The 8-week-old male ICR mice were killed according to the standard protocols of animal experiments of Fujian Medical University. The testes or epididymis were collected and measured. The testes and epididymis were incubated with $4 \%$ polyformaldehyde/PBS solution for $12 \mathrm{~h}$ and then dehydrated in $20 \%$ sucrose/PBS for $4 \mathrm{~h}$, and then in $30 \%$ sucrose/PBS for $4 \mathrm{~h}$. The samples were dissected into small pieces and embedded in Tissue-Tek O. C.T. compound (Sakura Cat. 4583). After incubated with liquid nitrogen for $2 \mathrm{~min}$, the samples were stored at $-80{ }^{\circ} \mathrm{C}$ for $12 \mathrm{~h}$. The $6-\mu \mathrm{m}$ cryo-sections were cut using a
Leica freezing microtome (Leica CM1860 UV) and then stored at $-80^{\circ} \mathrm{C}$ for subsequent analyses.

The cryo-sections were fixed with 4\% PFA/PBS for $10 \mathrm{~min}$, and then immersed in PBS solution three times for $3 \mathrm{~min}$. For permeation, the slides were incubated with $0.5 \%$ TritonX-100/PBS at room temperature for $10 \mathrm{~min}$. The slides were blocked with $1 \%$ BSA/PBST (0.05\% Tween 20 in PBS) for $1 \mathrm{~h}$. The specific primary antibody was diluted in $1 \%$ BSA/PBST and incubated with the samples at $4{ }^{\circ} \mathrm{C}$ for $12 \mathrm{~h}$. After washed by PBS for $15 \mathrm{~min}$, the samples were incubated with the secondary antibody at $37^{\circ} \mathrm{C}$ for $1 \mathrm{~h} .4^{\prime}, 6$-diamidino2-phenylindole (DAPI) (Beyotime Cat. C1006) were used for the staining of the nucleus. The slides were incubated with the anti-fading medium (Beyotime Cat. P1028S) and observed under a fluorescence microscope (Nikon Ti-S). The antibodies used in this study are listed as follows: the CENPE mouse monoclonal antibody (Santa Cruz Cat. SC-376685, 1:100), $\beta$-tubulin rabbit monoclonal antibody (Beyotime Cat. AF1216, 1:500), Alexa Fluor 488-labeled Goat Anti-Rabbit IgG $(\mathrm{H}+\mathrm{L})$ (Beyotime Cat. A0423, 1:500), Alexa Fluor 488labeled Goat Anti-Mouse IgG (H + L) (Beyotime Cat. A0428, 1:500), Alexa Fluor 555-labeled Donkey Anti-Rabbit IgG $(\mathrm{H}+\mathrm{L})$ (Beyotime Cat. A0453, 1:500), and Alexa Fluor 555labeled Donkey Anti-Mouse IgG $(\mathrm{H}+\mathrm{L})$ (Beyotime Cat. A0460, 1:500).

\section{Hematoxylin-eosin staining}

For Hematoxylin-eosin (HE) staining of testis cryo-sections, the slides were incubated with $70 \%$ ethanol for $2 \mathrm{~min}$, and then incubated with distilled water for $2 \mathrm{~min}$. After incubating with Mayer's hematoxylin solution for $5 \mathrm{~min}$, the slides were washed by running water for $5 \mathrm{~min}$. The samples were incubated with saturated lithium carbonate for $5 \mathrm{~s}$, and then incubated with distilled water for $2 \mathrm{~min}$. After incubating with $1 \%$ eosin for $1 \mathrm{~min}$, the samples were dehydrated by gradient ethanol and cleared in xylene for $5 \mathrm{~min}$. The samples were fixed with neutral resin for observation.

For HE staining of semen smear, semen was squeezed out of the epididymis, and then diluted using $0.9 \% \mathrm{NaCl}$. GSK923295 was added into $0.9 \% \mathrm{NaCl}$ to the final concentration as indicated. The sperms and GSK923295 were mixed and then incubated at $30^{\circ} \mathrm{C}$ for $4 \mathrm{~h}$. The slides were dried at room temperature for $10 \mathrm{~min}$. After incubating with 95\% ethanol for $10 \mathrm{~min}$, the slides were rinsed in distilled water for $3 \mathrm{~min}$. After incubating with Mayer's hematoxylin for $5 \mathrm{~min}$, the samples were incubated with ethanol hydrochloride for $30 \mathrm{~s}$. After staining by eosin for $1 \mathrm{~min}$, the samples were fixed by $95 \%$ ethanol for $5 \mathrm{~min}$. The slides were photographed by a light microscope (Nikon Ti-S) with a NA $\times 20 / 0.40$ objective and a NA $\times 40 / 0.75$ objective (Nikon).

\section{Flow cytometry}

For cell cycle analysis of spermatogenic cells, the testes were cut and digested with trypsin (Beyotime Cat. C0201) at 
$37^{\circ} \mathrm{C}$ for $15 \mathrm{~min}$. After washed twice in PBS for $3 \mathrm{~min}$, the cells were incubated with $75 \%$ ethanol for $12 \mathrm{~h}$. The cells were suspended in PBS and pelleted by centrifugation at $1200 \times g$. The samples were incubated with propidium iodide (Beyotime Cat. C1052) at $37^{\circ} \mathrm{C}$ for $2 \mathrm{~h}$. The flow cytometry was performed using a flow cytometer (BD, FACS Canto TM II).

\section{TUNEL assay}

For cell apoptosis analysis, the one step TUNEL apoptosis assay kit (Beyotime Cat. C1086) was used. The cells were fixed with $4 \%$ paraformaldehyde/PBS for $1 \mathrm{~h}$. After washing twice with PBS for $10 \mathrm{~min}$, the samples were incubated with $0.5 \%$ Triton X-100 in PBS for 5 min at room temperature. The samples were incubated with $\mathrm{TdT}$ solution (terminal deoxynucleotidyl transferase (TdT) and fluorescein isothiocyanate (FITC)-dUTP, $1: 10$ ) at $37^{\circ} \mathrm{C}$ for $1 \mathrm{~h}$. After washing three times with PBS, the slides were mounted with anti-fading medium and observed using a fluorescence microscope (Nikon Ti-S).

\section{Transmission electron microscopy}

For sample fixation: $3 \%$ glutaraldehyde, $1.5 \%$ polyformaldehyde in 0.1 M PBS (pH7.4) was used. After rinsing with $0.1 \mathrm{M}$ PBS, the samples were fixed using $1 \%$ osmium solution. After rinsing with $0.1 \mathrm{M}$ PBS, the samples were dehydrated using $30-90 \%$ gradient alcohol, $90-100 \%$ acetone, and then $100 \%$ acetone epoxy resin $(\mathrm{v} / \mathrm{v}, 1: 1)$ at room temperature for $2 \mathrm{~h}$. After incubating with epoxy resin at $35^{\circ} \mathrm{C}$ for $3 \mathrm{~h}$, the samples were incubated with epoxy resin at $35^{\circ} \mathrm{C}$ for $12 \mathrm{~h}$, at $45^{\circ} \mathrm{C}$ for $12 \mathrm{~h}$, at $60^{\circ} \mathrm{C}$ for 3 days. In all, $100 \mathrm{~nm}$ ultra-thin section were cut using an ultra-thin slicing machine (Leica EM UC-7). The samples were stained with uranium acetate for $20 \mathrm{~min}$. After rinsing with distilled water, the samples were stained using lead citrate for $5 \mathrm{~min}$. After rinsing with distilled water, the samples were imaged using an electron microscope (FEI, Tecnai G2). The quantification of chromatin arrangement and density was performed using a script from github (https://github.com/barouxlab/ ChromDensityNano) by the MATLAB software according to previous reported methods ${ }^{55}$.

\section{Image analyses and statistics}

All experiments were performed more than three times. The sample size was indicated in each figure legend. The investigator was blinded to the group allocation during the experiment and/or when assessing the outcome. The simple randomization was used in the sample analysis and animal experiments. Statistical analyses were conducted by the two-tailed unpaired Student's $t$ test using GraphPad Prism 6.0 software. Data were shown as mean \pm SEM. ns, $p>0.05$, "p $p<0.05, * " p<0.01, * * * p<0.001$, and ${ }^{* * * * *} p<0.0001$.

\section{Acknowledgements}

We sincerely thank all members of the Cytoskeleton Laboratory at Fujian Medical University for their help and discussions. This study was supported by the following grants: the Natural Science Foundation of Fujian Province, China (Grant No. 2019J05071), the Health and Family Planning Commission of Fujian Province, China (Grant No. 2018-1-69), Startup Fund for scientific research, Fujian Medical University (Grant No. 2017XQ1001), Fujian Medical University high level talents scientific research start-up funding project (Grant No. XRCZX2017025), and College Students' innovation and entrepreneurship training program (Grant No. 201810392035 and No. C19065).

\section{Author details}

'Department of Cell Biology and Genetics, The School of Basic Medical Sciences, Fujian Medical University, Fuzhou, Fujian 350122, China. ${ }^{2}$ Key Laboratory of Stem Cell Engineering and Regenerative Medicine, Fujian Province University, Fuzhou, Fujian 350122, China. ${ }^{3}$ Fujian Obstetrics and Gynecology Hospital, Fuzhou, Fujian 350001, China. ${ }^{4}$ Medical Research Center, Fujian Provincial Children's Hospital, Fujian Maternity and Child Health Hospital, Affiliated Hospital of Fujian Medical University, Fuzhou, Fujian 350001, China

\section{Data availability}

The data that support the findings of this study are available from the corresponding author upon reasonable request.

Conflict of interest

The authors declare that they have no conflict of interest.

\section{Publisher's note}

Springer Nature remains neutral with regard to jurisdictional claims in published maps and institutional affiliations.

The online version of this article (https://doi.org/10.1038/s41420-020-0261-8) contains supplementary material, which is available to authorized users.

Received: 22 January 2020 Revised: 4 March 2020 Accepted: 5 April 2020 Published online: 20 April 2020

\section{References}

1. Parra, M. T. et al. Sequential assembly of centromeric proteins in male mouse meiosis. PLoS Genet. 5, e1000417 (2009).

2. Lampson, M. A. \& Kapoor, T. M. The human mitotic checkpoint protein BubR1 regulates chromosome-spindle attachments. Nat. Cell Biol. 7, $93-98$ (2005).

3. Mao, Y., Desai, A. \& Cleveland, D. W. Microtubule capture by CENP-E silences BubR1-dependent mitotic checkpoint signaling. J. Cell Biol. 170, 873-880 (2005).

4. Silk, A. D. et al. Chromosome missegregation rate predicts whether aneuploidy will promote or suppress tumors. Proc. Natl Acad. Sci. USA 110, E4134-E4141 (2013).

5. Putkey, F. R. et al. Unstable kinetochore-microtubule capture and chromosomal instability following deletion of CENP-E. Dev. Cell 3, 351-365 (2002).

6. Yen, T. J. et al. CENP-E, a novel human centromere-associated protein required for progression from metaphase to anaphase. EMBO J. 10, 1245-1254 (1991).

7. Yen, T. J., Li, G., Schaar, B. T., Szilak, I. \& Cleveland, D. W. CENP-E is a putative kinetochore motor that accumulates just before mitosis. Nature 359, 536-539 (1992).

8. Espeut, J. et al. Phosphorylation relieves autoinhibition of the kinetochore motor Cenp-E. Mol. Cell. 29, 637-643 (2008).

9. Lawrence, C. J. et al. A standardized kinesin nomenclature. J. Cell Biol. 167, 19-22 (2004).

10. Liao, H., Li, G. \& Yen, T. J. Mitotic regulation of microtubule cross-linking activity of CENP-E kinetochore protein. Science 265, 394-398 (1994).

11. Chan, G. K. Schaar, B. T. \& Yen, T. J. Characterization of the kinetochore binding domain of CENP-E reveals interactions with the kinetochore proteins CENP- $F$ and hBUBR1. J. Cell Biol. 143, 49-63 (1998). 
12. Schaar, B. T., Chan, G. K., Maddox, P., Salmon, E. D. \& Yen, T. J. CENP-E function at kinetochores is essential for chromosome alignment. J. Cell Biol. 139, 1373-1382 (1997).

13. Yao, X., Abrieu, A., Zheng, Y., Sullivan, K. F. \& Cleveland, D. W. CENP-E forms a link between attachment of spindle microtubules to kinetochores and the mitotic checkpoint. Nat. Cell. Biol. 2, 484-2491 (2000).

14. McEwen, B. F. et al. CENP-E is essential for reliable bioriented spindle attachment, but chromosome alignment can be achieved via redundant mechanisms in mammalian cells. Mol. Biol. Cell. 12, 2776-2789 (2001).

15. Shrestha, R. L. \& Draviam, V. M. Lateral to end-on conversion of chromosomemicrotubule attachment requires kinesins CENP-E and MCAK. Curr. Biol. 23, 1514-1526 (2013).

16. Brown, K. D., Coulson, R. M., Yen, T. J. \& Cleveland, D. W. Cyclin-like accumulation and loss of the putative kinetochore motor CENP-E results from coupling continuous synthesis with specific degradation at the end of mitosis. J. Cell Biol. 125, 1303-1312 (1994).

17. Kumar, A., Kamaraj, B., Sethumadhavan, R. \& Purohit, R. Evolution driven structural changes in CENP-E motor domain. Interdiscip. Sci. 5, 102-111 (2013).

18. Gudimchuk, N. et al. Kinetochore kinesin CENP-E is a processive bi-directional tracker of dynamic microtubule tips. Nat. Cell Biol. 15, 1079-1088 (2013).

19. Kim, Y., Heuser, J. E., Waterman, C. M. \& Cleveland, D. W. CENP-E combines a slow, processive motor and a flexible coiled coil to produce an essential motile kinetochore tether. J. Cell Biol. 181, 411-419 (2008).

20. Sardar, H. S., Luczak, V. G., Lopez, M. M., Lister, B. C. \& Gilbert, S. P. Mitotic kinesin CENP-E promotes microtubule plus-end elongation. Curr. Biol. 20, 1648-1653 (2010).

21. Abrieu, A., Kahana, J. A., Wood, K. W. \& Cleveland, D. W. CENP-E as an essential component of the mitotic checkpoint in vitro. Cell 102, 817-826 (2000).

22. Garcia-Saez, I., Yen, T., Wade, R. H. \& Kozielski, F. Crystal structure of the motor domain of the human kinetochore protein CENP-E. J. Mol. Biol. $\mathbf{3 4 0}$ 1107-1116 (2004).

23. Guo, Y., Kim, C., Ahmad, S., Zhang, J. \& Mao, Y. CENP-E-dependent BubR1 autophosphorylation enhances chromosome alignment and the mitotic checkpoint. J. Cell Biol. 198, 205-217 (2012).

24. Maffini, S. et al. Motor-independent targeting of CLASPs to kinetochores by CENP-E promotes microtubule turnover and poleward flux. Curr. Biol. 19, 1566-1572 (2009).

25. Mao, Y., Abrieu, A. \& Cleveland, D. W. Activating and silencing the mitotic checkpoint through CENP-E-dependent activation/inactivation of BubR1. Cell 114, 87-98 (2003)

26. Weaver, B. A. et al. Centromere-associated protein- $E$ is essential for the mammalian mitotic checkpoint to prevent aneuploidy due to single chromosome loss. J. Cell. Biol. 162, 551-563 (2003).

27. Wood, K. W., Sakowicz, R., Goldstein, L. S. \& Cleveland, D. W. CENP-E is a plus end-directed kinetochore motor required for metaphase chromosome alignment. Cell 91, 357-366 (1997).

28. Tanudji, M. et al. Gene silencing of CENP-E by small interfering RNA in HeLa cells leads to missegregation of chromosomes after a mitotic delay. Mol. Biol. Cell. 15, 3771-3781 (2004).

29. Weaver, B. A., Silk, A. D. \& Cleveland, D. W. Cell biology: nondisjunction, aneuploidy and tetraploidy. Nature 442, E9-E10 (2006).

30. Barisic, M., Aguiar, P., Geley, S. \& Maiato, H. Kinetochore motors drive congression of peripheral polar chromosomes by overcoming random armejection forces. Nat. Cell Biol. 16, 1249-1256 (2014).

31. Parra, M. T. et al. Expression and behaviour of CENP-E at kinetochores during mouse spermatogenesis. Chromosoma 111, 53-61 (2002).

32. Radford, S. J. et al. Lateral and end-on kinetochore attachments are coordinated to achieve bi-orientation in Drosophila oocytes. PLoS Genet. 11, e1005605 (2015).

33. Balamuth, N. J. et al. Serial transcriptome analysis and cross-species integration identifies centromere-associated protein $\mathrm{E}$ as a novel neuroblastoma target. Cancer Res. 70, 2749-2758 (2010).
34. Qian, X. et al. Discovery of the first potent and selective inhibitor of centromere-associated protein E: GSK923295. ACS Med. Chem. Lett. 1, 30-34 (2010).

35. Wood, K. W. et al. Antitumor activity of an allosteric inhibitor of centromere-associated protein-E. Proc. Natl Acad. Sci. USA 107, 5839-5844 (2010).

36. Wolkowicz, M. J. et al. Refinement of the differentiated phenotype of the spermatogenic cell line GC-2spd(ts). Biol. Reprod. 55, 923-932 (1996).

37. Cooke, C. A., Schaar, B., Yen, T. J. \& Earnshaw, W. C. Localization of CENP$E$ in the fibrous corona and outer plate of mammalian kinetochores from prometaphase through anaphase. Chromosoma 106, 446-455 (1997).

38. Brown, K. D., Wood, K. W. \& Cleveland, D. W. The kinesin-like protein CENP-E is kinetochore-associated throughout poleward chromosome segregation during anaphase-A. J. Cell Sci. 109, 961-969 (1996).

39. Kallio, M., Mustalahti, T., Yen, T. J. \& Lähdetie, J. Immunolocalization of alphatubulin., gamma-tubulin, and CENP-E in male rat and male mouse meiotic divisions: pathway of meiosis I spindle formation in mammalian spermatocytes. Dev. Biol. 195, 29-37 (1998).

40. Duesbery, N. S. et al. CENP-E is an essential kinetochore motor in maturing oocytes and is masked during mos-dependent., cell cycle arrest at metaphase II. Proc. Natl Acad. Sci. USA 94, 9165-9170 (1997).

41. Chan, G. K. et al. Human BUBR1 is a mitotic checkpoint kinase that monitors CENP-E functions at kinetochores and binds the cyclosome/APC. J. Cell Biol. 146, 941-954 (1999).

42. Akera, T. et al. Mad1 promotes chromosome congression by anchoring a kinesin motor to the kinetochore. Nat. Cell Biol. 17, 1124-1133 (2015).

43. Maia, A. F. et al. Aurora B kinase cooperates with CENP-E to promote timely anaphase onset. Chromosoma 119, 405-413 (2010).

44. Yucel, J. K. et al. CENP-meta, an essential kinetochore kinesin required for the maintenance of metaphase chromosome alignment in Drosophila. J. Cell Biol. 150, 1-11 (2000).

45. Lombillo, V. A., Nislow, C., Yen, T. J., Gelfand, V. I. \& Mclntosh, J. R. Antibodies to the kinesin motor domain and CENP-E inhibit microtubule depolymerization-dependent motion of chromosomes in vitro. J. Cell Biol. 128, 107-115 (1995).

46. Kapoor, T. M. et al. Chromosomes can congress to the metaphase plate before biorientation. Science 311, 388-391 (2006).

47. Yardimci, H., van Duffelen, M., Mao, Y., Rosenfeld, S. S. \& Selvin, P. R. The mitotic kinesin CENP-E is a processive transport motor. Proc. Natl Acad. Sci. U.S.A. 105 6016-6021 (2008).

48. Zhang, $\mathrm{H}$. et al. Optogenetic control of kinetochore function. Nat. Chem. Biol. 13, 1096-1101 (2017).

49. Shastry, S. \& Hancock, W. O. Interhead tension determines processivity across diverse N-terminal kinesins. Proc. Natl Acad. Sci. U.S.A. 108, 16253-16258 (2011).

50. Nousiainen, M., Silljé, H. H., Sauer, G., Nigg, E. A. \& Körner, R. Phosphoproteome analysis of the human mitotic spindle. Proc. Natl Acad. Sci. U.S.A. 103 5391-5396 (2006)

51. Liu, D. et al. Human NUF2 interacts with centromere-associated protein E and is essential for a stable spindle microtubule-kinetochore attachment. J. Biol. Chem. 282, 21415-21424 (2007).

52. Vitre, B. et al. Kinetochore-microtubule attachment throughout mitosis potentiated by the elongated stalk of the kinetochore kinesin CENP-E. Mol. Biol. Cell 25, 2272-2281 (2014).

53. Gui, L. \& Homer, H. Spindle assembly checkpoint signalling is uncoupled from chromosomal position in mouse oocytes. Development 139, 1941-1946 (2012).

54. Pellman, D. Cell biology: aneuploidy and cancer. Nature 446, 38-39 (2007).

55. Cherkezyan, L. et al. Nanoscale changes in chromatin organization represent the initial steps of tumorigenesis: a transmission electron microscopy study. BMC Cancer 14, 189 (2014). 